\title{
Multiphase transport model for relativistic nuclear collisions
}

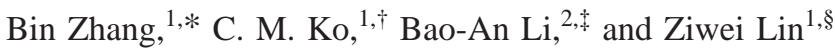 \\ ${ }^{1}$ Cyclotron Institute and Physics Department, Texas A\&M University, College Station, Texas 77843 \\ ${ }^{2}$ Department of Chemistry and Physics, Arkansas State University, P.O. Box 419, State University, Arkansas 72467-0419
}

(Received 7 December 1999; published 1 May 2000)

\begin{abstract}
To study heavy ion collisions at energies available from the Relativistic Heavy Ion Collider (RHIC), we have developed a multiphase transport model that includes both initial partonic and final hadronic interactions. Specifically, the Zhang's parton cascade (ZPC) model, which uses as input the parton distribution from the heavy ion jet interaction generator (HIJING) model, is extended to include the quark-gluon-to-hadronicmatter transition and also final-state hadronic interactions based on a relativistic transport (ART) model. Predictions of the model for central $\mathrm{Au}$ on $\mathrm{Au}$ collisions at RHIC are reported.
\end{abstract}

PACS number(s): 25.75.-q, 24.10.Lx, 24.10.Jv

The beginning of experiments at the Relativistic Heavy Ion Collider (RHIC) this year will start an exciting new era in nuclear and particle physics. The estimated high energy density in central heavy ion collisions at RHIC is expected to lead to the formation of a large region of deconfined matter of quarks and gluons, the quark gluon plasma (QGP). This would give us the opportunity to study the properties of the QGP and its transition to hadronic matter, which would then shed light on the underlying fundamental theory of strong interactions, quantum chromodynamics (QCD).

Because of the complexity of heavy ion collision dynamics, Monte Carlo event generators are needed to relate the experimental observations to the underlying theory. This has already been shown to be the case in heavy ion collisions at existing accelerators such as the SIS, AGS, and SPS [1-6]. As minijet production is expected to play an important role at RHIC energies [7], models for partonic transport have been studied $[8,9]$. Furthermore, transport models that include both partonic and hadronic degrees of freedom are being developed $[10,11]$. We have recently also developed such a multiphase transport (AMPT) model. It starts from initial conditions that are motivated by perturbative QCD and incorporates the subsequent partonic and hadronic spacetime evolution. In particular, we have used the heavy ion jet interaction generator (HIJING) model [7] to generate the initial phase space distribution of partons and the Zhang's parton cascade (ZPC) model [9] to follow their rescatterings. A modified HIJING fragmentation scheme is then introduced for treating the hadronization of the partonic matter. The evolution of the resulting hadron system is treated in the framework of a relativistic transport (ART) model [2]. In this paper, we shall describe this new multiphase transport model and show its predictions for central Au-on-Au collisions at RHIC.

In the AMPT model, the initial parton momentum distribution is generated from the HIJING model, which is a Monte Carlo event generator for hadron-hadron, hadron-

\footnotetext{
*Electronic address: bzhang@kopc1.tamu.edu

†Electronic address: ko@comp.tamu.edu

‡Electronic address: bali@navajo.astate.edu

${ }^{\S}$ Electronic address: lin@kopc1.tamu.edu
}

nucleus, and nucleus-nucleus collisions. The HIJING model treats a nucleus-nucleus collision as a superposition of binary nucleon-nucleon collisions. For each pair of nucleons, the impact parameter is determined using the nucleon transverse positions generated from a Woods-Saxon nuclear density distribution. The eikonal formalism is then used to determine the probability for a collision to occur. For a given collision, one further determines if it is an elastic or inelastic collision, a soft or hard inelastic interaction, and the number of jets produced in a hard interaction. To take into account nuclear effects in hard interactions, an impact parameter-dependent parton distribution function based on the Mueller-Qiu parametrization [12] of nuclear shadowing is used. Afterwards, PYTHIA routines [13] are called to describe hard interactions, while soft interactions are treated according to the Lund model [14].

In the HIJING model, minijets from produced partons are quenched by losing energy to the wounded nucleons close to their straight-line trajectories. In the AMPT model, we replace the parton quenching by their rescatterings. To generate the initial phase space distribution for the parton cascade, the formation time for each parton is determined according to a Lorentzian distribution with a half width $t_{f}=E / m_{T}^{2}$ [15], where $E$ and $m_{T}$ are the parton energy and transverse mass, respectively. Positions of formed partons are calculated from those of their parent nucleons using straight-line trajectories. During the time of formation, partons are considered to be part of the coherent cloud of parent nucleons, and they thus do not suffer rescatterings.

The parton cascade in the AMPT model is carried out using the ZPC model [9]. At present, this model includes only gluon-gluon scatterings with cross sections taken to be the leading divergent cross section regularized by a medium generated screening mass. The latter is related to the phase space density of produced partons [16]. For simplicity, a constant screening mass of $\mu=3 \mathrm{fm}^{-1}$ is used in the present study. The neglect of quark scatterings is not expected to affect appreciablly the collision dynamics because their number in heavy ion collisions at collider energies is much smaller than that of gluons [17] and their scattering cross sections are also much smaller than that between gluons [18].

Once partons stop interacting, they are converted into hadrons using the HIJING fragmentation scheme after an 


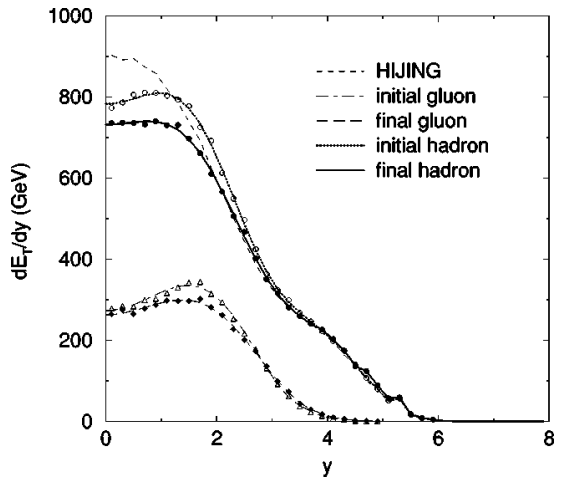

FIG. 1. Transverse energy rapidity distribution for central $(b$ $=0) \mathrm{Au}+\mathrm{Au}$ collisions at RHIC.

additional proper time of about $1.2 \mathrm{fm}$. In the default HIJING model, a diquark is treated as a single entity, and this leads to an average rapidity shift of about one unit in the net baryon distribution. We modify this fragmentation scheme to allow the formation of diquark-antidiquark pairs. With a fragmentation probability of $80 \%$ for $B M \bar{B}$ and $20 \%$ for $B \bar{B}$ from the diquark-antidiquark pairs, we have obtained a reasonable description of the measured net baryon rapidity distribution in $\mathrm{Pb}+\mathrm{Pb}$ collisions at $158 \mathrm{GeV} /$ nucleon [19].

For the evolution of hadrons, we use the ART model, which is a successful hadronic transport model for heavy ion collisions at AGS energies. To extend the model to heavy ion collisions at RHIC, we have further included nucleonantinucleon annihilation channels, the inelastic interactions of kaons and antikaons, and neutral kaon production. In the ART model, multiparticle production is modeled through the formation of resonances. Since the inverse double resonance channels have smaller cross sections than those calculated directly from the detailed balance, we thus adjust the double resonance absorption cross sections to fit the NA49 data [19].

In Fig. 1, we show the rapidity distribution of transverse energy in $\mathrm{Au}+\mathrm{Au}$ central $(b=0)$ collisions at RHIC. The open triangles give the gluon $d E_{T} / d y$ from the HIJING model. It is the transverse energy produced from converting the initial parton longitudinal momenta into the transverse direction during the passage of two colliding nuclei. Since the longitudinal expansion of the resulting partonic matter develops much earlier than the transverse expansion, the parton longitudinal momenta are expected to decrease in the local rest frame. However, parton scatterings would convert part of the transverse momentum back to the longitudinal direction [20], leading to a decrease of $d E_{T} / d y$ by about $15 \mathrm{GeV}$ as shown by the difference between the final (solid triangles) and the initial $d E_{T} / d y$ distributions. Including the hadronic evolution further reduces $d E_{T} / d y$ by about $50 \mathrm{GeV}$. Because of jet quenching and a different fragmentation scheme, the default HIJING is shown to give a higher $d E_{T} / d y$ than the AMPT model at central rapidities. We note that the perturbatively produced gluons account for a significant fraction (about 1/3) of the total $d E_{T} / d y$ and that both the partonic and the hadronic evolution contribute appreciably to the longitudinal work.

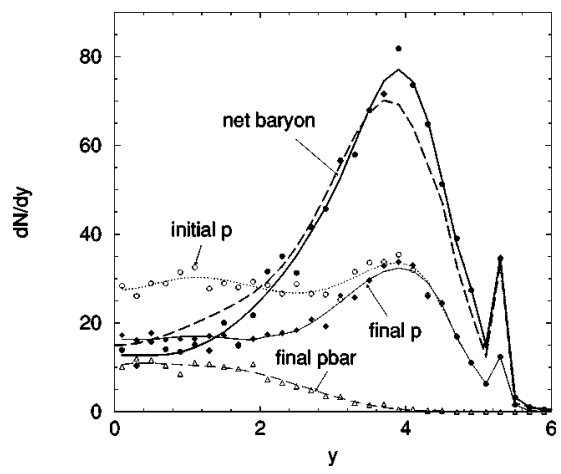

FIG. 2. Baryon rapidity distributions for RHIC $\mathrm{Au}+\mathrm{Au}$ central ( $b=0)$ collisions. The thick solid line represents the default AMPT net baryon distribution and the thick dashed line gives the result when nuclear shadowing is turned off.

Figure 2 shows the baryon rapidity distributions. It is seen that the net baryon distribution from the AMPT model has a peak value of 80 at $y \sim 3.9$ while that from the default HIJING model has a peak value of 85 at $y \sim 4.5$. The larger rapidity shift in the AMPT model is due to the modified fragmentation of diquarks. At central rapidity, the AMPT model predicts a net baryon number of about 12, which is similar to that from the default HIJING model. Since only baryons and antibaryons with similar rapidities can annihilate as a result of boot invariance, the net baryon distribution is not much affected by hadronic rescatterings. Many antiprotons (about 50\%) are seen to survive the absorption in hadronic matter, leading to a value of about 10 at central rapidities. The $\bar{p} / p$ ratio at central rapidity is about $60 \%$, which is much larger than the $10 \%$ seen in $\mathrm{Pb}+\mathrm{Pb}$ collisions at $158 \mathrm{GeV} /$ nucleon at SPS [21].

The final meson rapidity distribution is shown in Fig. 3. The prediction from the AMPT model has a distinctive plateau structure around central rapidities. Results using the default HIJING model show instead a peak at central rapidity with a higher rapidity density. Also shown in the figure is the distribution of kaons produced from both string fragmentation and hadronic interactions. The latter is seen to enhance significantly the kaon yield.

The transverse mass distributions of protons, pions, and kaons in the rapidity range of $(-1,1)$ are shown in Fig. 4.

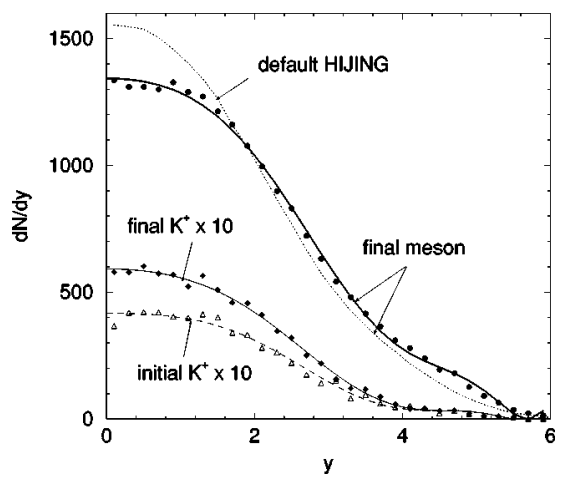

FIG. 3. Meson rapidity distributions for RHIC $\mathrm{Au}+\mathrm{Au}$ central $(b=0)$ collisions. 


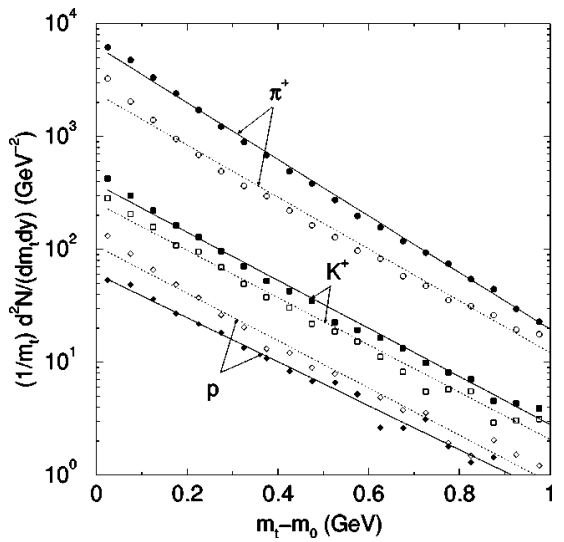

FIG. 4. Hadron transverse mass distributions for RHIC $\mathrm{Au}+\mathrm{Au}$ central $(b=0)$ collisions. Open and solid symbols correspond to the initial and final distributions, respectively.

Their inverse slope parameters can be determined from exponential regression fits to the calculated results, shown by the solid and dotted lines. Immediately after hadronization, they are $189 \mathrm{MeV}, 207 \mathrm{MeV}$, and $207 \mathrm{MeV}$, respectively, for $\pi^{+}$'s, $K^{+}$'s, and protons. After hadronic rescatterings, the inverse slope parameter of $\pi^{+}$'s drops to $173 \mathrm{MeV}$ while their number increases due to the decay of unstable hadrons. The inverse slope parameter of $K^{+}$'s is about $204 \mathrm{MeV}$ after rescatterings and thus remains essentially unchanged. For protons, hadronic rescatterings increase moderately the inverse slope parameter of their transverse momentum distribution to a value of $223 \mathrm{MeV}$. Our results thus indicate that only a weak radial flow is developed in heavy ion collisions at RHIC.

We have checked that the above results are hardly modified when the parton formation time or the parton-parton cross section is increased by a factor of 2 . The latter is achieved by varying the parton screening mass. This is understandable as the hadronic observables discussed in the above are sensitive to the angle-weighted parton cross section, which does not change much by varying the parton screening mass. If the parton screening mass is reduced, the total cross section increases but becomes more forward peaked. Similarly, increasing the parton screening mass reduces the total cross section which, however, becomes more isotropic. As to the parton formation time, its magnitude is small (about a tenth of a $\mathrm{fm} / \mathrm{c}$ ) compared to the mean free path of partons (about $1 \mathrm{fm} / c$ ), so reasonable variations of its value does not change the final hadronic observables.

A major uncertainty in our model is the treatment of nuclear shadowing effects on the initial parton distribution. To study this uncertainty, we have turned off nuclear shadowing in the HIJING model. This leads to a $50 \%$ increase of the gluon density in the central rapidity. As a result, the final gluon $d E_{T} / d y$ increases to $390 \mathrm{GeV}$ and the final hadron $d E_{T} / d y$ and $d N / d y$ increase to $960 \mathrm{GeV}$ and 1730 , respectively. The decrease of $d E_{T} / d y$ during the partonic and hadronic scatterings is a good measure of the longitudinal work done by the system. In the partonic stage, it is $15 \mathrm{GeV}$ with default nuclear shadowing and increases to $30 \mathrm{GeV}$ without shadowing. The corresponding values in the hadronic stage

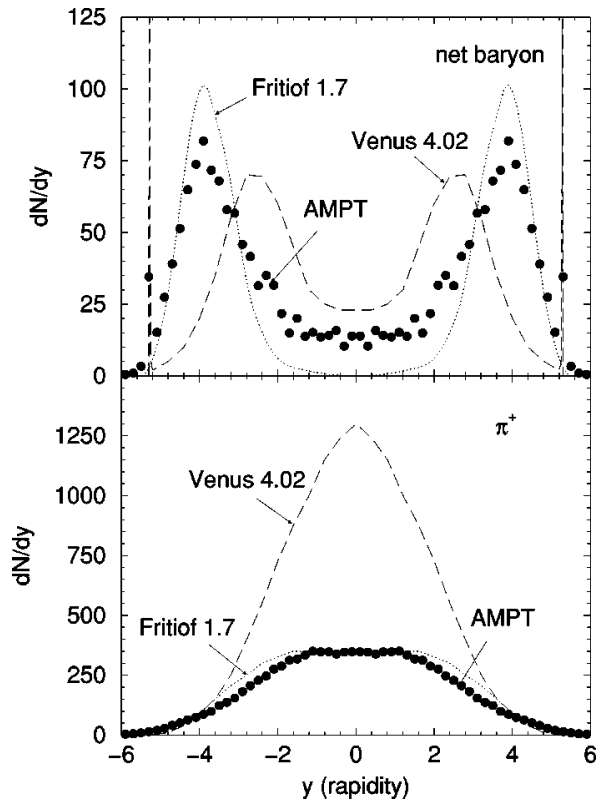

FIG. 5. Comparisons of AMPT, FRITIOF1.7, and VENUS4.02 predictions for RHIC $\mathrm{Au}+\mathrm{Au}$ central $(b=0)$ collisions.

are $50 \mathrm{GeV}$ and $70 \mathrm{GeV}$, respectively. The relative increase in the hadronic stage is smaller as a result of the increasing importance of the transverse expansion. Without nuclear shadowing, there are more particles produced in the central rapidity region, leading thus to an enhanced production of diquark-antidiquark pairs and hence a slightly larger rapidity shift of the net baryon distribution as shown by the thick dashed curve in Fig. 2. Both strangeness production and radial flow are also increased in the absence of nuclear shadowing. In particular, the $K^{+}$central rapidity densities are now 50 and 80 before and after hadronic scatterings instead of 40 and 60 in the case with nuclear shadowing. Also, the final proton slope parameter is $223 \mathrm{MeV}$ with default shadowing but increases to $238 \mathrm{MeV}$ without nuclear shadowing. Our results thus show that both initial nuclear shadowing and final-state interactions are important in heavy ion collisions at RHIC energies. We note that the importantance of the nuclear shadowing effect has also been pointed out in Ref. [22].

In Fig. 5, the AMPT results are compared with the predictions [23] from two widely used models, the FRITIOF1.7 [24] and the VENUS4.02 [25]. The upper panel shows the comparison of net baryon rapidity distributions. While the FRITIOF1.7 and the AMPT model give similar peak rapidity values, the VENUS4.02 has a smaller peak rapidity value, i.e., a larger net baryon rapidity loss. This is due to the different diquark breaking mechanism used in the vENUS4.02. Since the wee parton distribution $(P(x \pm)=1 / x \pm)$ is used in the FRITIOF1.7 to describe the soft momentum transfer processes, it leads to a zero baryon density in central rapidities, which is different from those from HIJING model and VENUS4.02 that are based on the dual parton model. The lower panel of Fig. 5 shows the $\pi^{+}$rapidity distribution. Since a statistical model is used in the VENUS model to treat the hadronization of a parton droplet, it gives a larger peak of final $\pi^{+}$distri- 
bution in the central rapidities than the FRITIOF and HIJING. Because of a different treatment of the soft momentum transfer processes, the FRITIOF gives a wider rapidity distribution than the AMPT model. The forthcoming RHIC data will allow one to test the different predictions from these models.

In conclusion, we have developed for heavy ion collisions at the Relativistic Heavy Ion Collider a multiphase transport model that includes both partonic and hadronic evolution. The model shows that both partons and hadrons contribute to the longitudinal collective work. Because of the production of diquark-antidiquark pairs, there is a relatively large rapidity shift of net baryons compared to the default HIJING fragmentation scheme. Many antiprotons survive final-state interactions and are expected to be observed at RHIC. Also, our model gives a wider meson rapidity plateau at central rapidities than the prediction from the default HIJING model. Furthermore, kaon production is enhanced appreciably due to the production from hadronic interactions. A moderate increase of the inverse slope parameter of the proton transverse momentum distribution is also seen, indicating the development of a weak radial flow as a result of hadronic rescatterings.

The AMPT model in its present form has not included elastic quark scatterings and inelastic parton scatterings. Although elastic quark scatterings are not expected to be important, inelastic parton scatterings would increase the energy loss [7] and enhance strangeness production [26]. In the future, we shall study how the inclusion of parton inelastic scatterings and also using different hadronization schemes would affect the results obtained here. Furthermore, we shall compare our predictions with the experimental data soon to be available from RHIC in order to better understand the collision dynamics.

The authors thank X.N. Wang and K. Werner for helpful discussions. This work was supported by the National Science Foundation under Grant No. PHY-9870038, Welch Foundation Grant No. A-1358, and Texas Advanced Research Grant No. FY97-010366-068.
[1] C.M. Ko and G.Q. Li, J. Phys. G 22, 1673 (1996).

[2] B.A. Li and C.M. Ko, Phys. Rev. C 52, 2037 (1995).

[3] S.H. Kahana, D.E. Kahana, Y. Pang, and T.J. Schlagel, Ann. Phys. (San Diego) 46, 31 (1996).

[4] W. Cassing and E.L. Bratkovskaya, Phys. Rep. 308, 65 (1999).

[5] H. Sorge, Phys. Rev. C 52, 3291 (1995).

[6] S.A. Bass et al., Prog. Part. Nucl. Phys. 42, 313 (1999).

[7] X.N. Wang and M. Gyulassy, Phys. Rev. D 44, 3501 (1991); M. Gyulassy and X.N. Wang, Comput. Phys. Commun. 83, 307 (1994).

[8] K. Geiger, Comput. Phys. Commun. 104, 70 (1997).

[9] B. Zhang, Comput. Phys. Commun. 109, 193 (1998); 111, 276(E) (1998).

[10] Y. Nara, Nucl. Phys. A638, 555c (1998).

[11] S.A. Bass, M. Hofmann, M. Bleicher, L. Bravina, E. Zabrodin, H. Stoecker, and W. Greiner, Phys. Rev. C 60, 021901 (1999).

[12] A.H. Mueller and J. Qiu, Nucl. Phys. B268, 427 (1986); ibid. B291, 746 (1987).

[13] T. Sjöstrand, Comput. Phys. Commun. 82, 74 (1994).

[14] B. Andersson, G. Gustafson, and B. Nilsson-Almquist, Nucl. Phys. B281, 289 (1987).

[15] M. Gyulassy and X.N. Wang, Nucl. Phys. B420, 583 (1994); X.N. Wang, M. Gyulassy, and M. Plümer, Phys. Rev. D 51,
3436 (1995).

[16] T.S. Biró, B. Müller, and X.N. Wang, Phys. Lett. B 283, 171 (1992).

[17] K.J. Eskola, Nucl. Phys. A590, 383c (1995).

[18] E. Shuryak, Phys. Rev. Lett. 68, 3270 (1992).

[19] B. Zhang, C.M. Ko, B.A. Li, and Z. Lin, in Proceedings of Relativistic Heavy Ion Mini-symposium in APS Centennial Meeting.

[20] M. Gyulassy, Y. Pang, and B. Zhang, Nucl. Phys. A626, 999 (1997).

[21] NA44 Collaboration, N. Xu et al. Nucl. Phys. A610, 175c (1996).

[22] X. N. Wang's contribution in S. Bass et al., Nucl. Phys. A661, 205 (1999).

[23] BRAHMS Collaboration, D. Beavis et al., in Proceedings of the Pre-Conference Workshop, Eleventh International Conference on Ultra-Relativistic Nucleus-Nucleus Collisions, 1995, p. 11; BRAHMS Collaboration, B. Moskowitz et al., ibid., p. 21.

[24] B. Nilsson-Almqvist and E. Stenlund, Comput. Phys. Commun. 43, 387 (1987); H. Pi, ibid. 71, 173 (1992).

[25] K. Werner, Phys. Rep. 232, 87 (1993).

[26] K. Geiger, Phys. Rep. 258, 237 (1995). 